\title{
Social Vigilance of Friends and Foes in Western Lowland Gorillas (Gorilla gorilla gorilla)
}

\author{
Tom S. Roth ${ }^{1,2,3, *} \&$ Elisabeth H.M. Sterck ${ }^{1,4}$ \\ ${ }^{1}$ Animal Ecology, Utrecht University, Utrecht, The Netherlands \\ ${ }^{2}$ Apenheul Primate Park, Apeldoorn, The Netherlands \\ ${ }^{3}$ Institute of Psychology, Cognitive Psychology Unit, Leiden University, Leiden, The Netherlands \\ ${ }^{4}$ Ethology Research, BPRC, Rijswijk, The Netherlands \\ *Corresponding author (Email: tsroth1993@gmail.com)
}

Citation - Roth, T. S., \& Sterck, E. H. M. (2020). Social vigilance of friends and foes in western lowland gorillas (Gorilla gorilla gorilla). Animal Behavior and Cognition, 7(4), 537-555.

doi: https://doi.org/10.26451/abc.07.04.06.2020

\begin{abstract}
In social species, such as many primate species, conspecifics can pose a threat and individuals that are socially vigilant can prevent harassment. Many previous studies have focused on the role of agonistic interactions on social vigilance. In a variety of primate species, individuals are more vigilant for aggressive or dominant group members. In contrast, only few studies have investigated whether affiliative relationships also affect social vigilance. These studies revealed that individuals with an affiliative relationship showed lower levels of vigilance towards each other. Here, we tested the differential effects of both dyadic agonism and affiliation on the level of social vigilance of group-living western lowland gorillas (Gorilla gorilla gorilla) at Apenheul Primate Park, The Netherlands. We made continuous focal observations of agonistic and affiliative interactions and we scored level of vigilance during neutral approaches of conspecifics. We found that dyads with many affiliative interactions showed lower levels of vigilance towards each other. The opposite pattern was found for agonistic experiences, but this effect was not statistically robust. In addition, the adult male and adolescent males received higher levels of social vigilance than individuals from other age-sex classes. Our results indicate that level of social vigilance was linked to affiliative and, to a lesser extent, agonistic relationships in western lowland gorillas. We suggest that future studies in both egalitarian and despotic species should investigate whether, next to aggression, affiliation also influences social vigilance.
\end{abstract}

Keywords - Social attention, Despotism, Good relationships, Friendship, Apes, Social complexity

Vigilance behavior can be linked to benefits and costs of group living. Traditionally, research on animal vigilance focused on predator vigilance (general: Beauchamp, 2015; Elgar, 1989; primates: Allan $\&$ Hill, 2018; Treves, 2000). Individual time spent on vigilance can be reduced in larger groups since vigilance by other group members enhances combined predator detection ability (Elgar, 1989; Treves, 2000). However, research on primates does not conform with this hypothesis, as many studies have not found a negative relationship between group size and vigilance (Treves, 2000), despite that safety against predators forms an important benefit of group living in primates (e.g., Bettridge \& Dunbar, 2012; van Schaik \& van Noordwijk, 1985). This unexpected outcome may be the result of social vigilance: primates are vigilant to detect and monitor conspecifics rather than only monitoring predators (Treves, 2000). Accordingly, common marmosets (Callithrix jacchus) display less antipredator vigilance and more social vigilance in larger groups, suggesting that the group size effect on vigilance works in opposite directions 
for antipredator vigilance and social vigilance (Gosselin-Ildari \& Koenig, 2012). How social vigilance is affected by previous experiences with group members is still unclear. However, social vigilance may be modulated by agonistic and affiliative experiences of individuals.

Social vigilance is especially important when individuals are confronted with social costs of group living, such as within-group competition for resources (Aureli et al., 2002; van Schaik, 1983). Within-group competition results in conflict between group members and may lead to physical harm (e.g., Drews, 1996), recurrent aggression (e.g., Aureli, 1992) and anxiety (e.g., Maestripieri et al., 1992). Because of these consequences, behavioral mechanisms that avoid or reduce aggression, its severity or consequences are an important evolutionary prerequisite for group living (de Waal, 2000). One way to do this is by keeping an eye on more dominant or aggressive group members (e.g., Pannozzo et al., 2007), so that individuals can avoid them before a conflict starts (Evers et al., 2012). Simultaneously, group living has also social benefits since group members benefit from their social relationships (primates: Ellis et al., 2019; Massen et al., 2010; Seyfarth \& Cheney, 2012; Silk, 2003; Silk et al., 2010a,b; cetaceans: Connor, 2007; Ellis et al., 2017; horses: Cameron et al., 2009). Such good relationships may also relax social vigilance (Kutsukake, 2006; Watts, 1998). Thus, both affiliative and agonistic experiences may affect levels of social vigilance.

In conceptual models of social relationships, aggression and affiliation are often considered two opposite ends on a gradient (Thompson, 2019). However, evidence in primates shows that they are largely separate dimensions. Aggression rates of individuals with affiliative and non-affiliative relationships are often relatively similar in despotic species (e.g., rhesus macaques, Macaca mulatta: Bernstein \& Ehardt, 1986; Widdig et al., 2002; chacma baboons, Papio hamadryas ursinus: Silk et al., 2010a, African green monkeys, Chlorocebus aethiops: Chalyan \& Meishvili, 2007; review: Bernstein, 1991; Silk, 2002) and possibly in more egalitarian species as well (e.g., western lowland gorilla, Gorilla gorilla gorilla: Stokes, 2004). Therefore, it is important to consider the effects of agonistic interactions and dyadic affiliation separately.

Multiple studies have investigated the effect of agonistic experiences on social vigilance. These studies have shown that subordinate individuals spend more time on social vigilance (talapoin monkeys, Miopithecus talapoin: Keverne et al., 1978; patas monkeys, Erythrocebus patas: McNelis \& BoatrightHorowitz, 1998; capuchin monkeys, Cebus apella: Pannozzo et al., 2007; blue monkeys, Cercopithecus mitis: Gaynor \& Cords, 2012). In addition, dominant individuals are more often the target of vigilance than subordinates (talapoin monkeys: Keverne et al., 1978; patas monkeys: McNelis \& BoatrightHorowitz, 1998; capuchin monkeys: Pannozzo et al., 2007; mandrills, Mandrillus sphinx: Schino \& Sciarretta, 2016). Given these results, it seems that agonistic experiences considerably modulate social vigilance in primates.

While early conceptual models have mostly focused on the role of aggression, the importance of affiliative interactions in primate sociality has recently been emphasized as well (Overduin-de Vries et al., 2020; Sussman et al., 2005; Vermande \& Sterck, in press). Possibly, such affiliative interactions can also influence social vigilance. However, so far only two studies have incorporated dyadic affiliation in the study of social vigilance (chimpanzees, Pan troglodytes: Kutsukake, 2006; mountain gorillas, Gorilla beringei beringei: Watts, 1998). Both studies found a relation between affiliative behavior and level of vigilance: individuals spent less time on monitoring affiliative conspecifics (Kutsukake, 2006) or showed lower levels of vigilance during their approaches (Watts, 1998). These previous results corroborate the idea that social vigilance might be modulated by affiliative experiences.

We investigated (1) how dyadic aggression and affiliation were related, and (2) whether levels of social vigilance are explained by dyadic agonistic experiences and dyadic affiliation in western lowland gorillas. Note that these two explanations are not mutually exclusive. Western lowland gorillas live in family groups consisting of one adult silverback male, multiple adult females, and their offspring (Magliocca et al., 1999). Females have relatively egalitarian relationships and generally show both low rates of aggression and low rates of affiliation (Stokes, 2004). In addition, western lowland gorilla females do not form a linear dominance hierarchy (Scott \& Lockard, 1999), and show little to no 
submissive behaviors (Stokes, 2004). We observed a naturalistic family group of western lowland gorillas housed in Apenheul Primate Park, The Netherlands, and measured their reactions to approaching conspecifics. Our study is one of few that analyzes both the effects of agonism and affiliation on social vigilance, thereby allowing a comparison of their separate effects.

\section{Method}

\section{Subjects and Housing}

We studied a family group of western lowland gorillas (Gorilla gorilla gorilla) in Apenheul Primate Park (Apeldoorn, The Netherlands) in 2016-2017. At the start of the study, the group consisted of 14 animals: 1 adult male, 5 adult females, 2 adolescent males, 1 adolescent female, 3 juvenile females and 2 juvenile males (Appendix, Table A1). The silverback male, Jambo, had lived with the other adult individuals in the group since his introduction in 2006. All adolescent and juvenile individuals were his offspring. The group size was similar to wild groups and the number of individuals from each age-sex class fell within the range of wild western lowland gorillas (Magliocca et al., 1999). The group encompassed four matrilines. The only individual without maternal relatives was the adult male. One female, Kisiwa, was moved to another zoo halfway the observation period.

The group normally had access to a large 1.1-hectare outdoor enclosure. However, during the study the gorillas were housed in a $201-\mathrm{m}^{2}$ indoor enclosure, as the outdoor island was being reconstructed. Because the park was closed during the winter, no visitors were present in the inside enclosure during the observation period.

\section{Data Collection and Ethogram}

Data were collected between December 2016 and March 2017 by TSR. Observations were conducted in 4 timeslots: 9:00-11:00, 11:00-13:00, 13:00-15:00 and 15:00-17:00. Continuous focal samples of 15 min were made for all individuals (Altmann, 1974), one at a time. Each individual was observed at least once in each timeslot every week, resulting in approximately 9 hrs of observations for each individual (Appendix, Table A1). The observations of every individual were equally spread among timeslots. Focal observations were collected using the software system Noldus The Observer XT 11 (Noldus, 1991).

The ethogram that was used for this study (Appendix, Table A2) included both duration and point behaviors and was a combination of two already existing ethograms for western lowland gorillas (Less et al., 2010; Sarfaty et al., 2012). First, the time budget state of the individual was scored (foraging, inactive, move, social play, allogrooming, other; Table A2). Time budget states were mutually exclusive and a focal individual was assigned one of the time budget states at all time during the observation. Apart from the time budget states Social Play and Allogrooming, the ethogram contained two affiliative substates, namely Proximity and Contact Sitting. Proximity and Contact Sitting were substates of the time budget behaviors Inactive and Foraging. These substates, together with the states Social Play and Grooming, were used to characterize the affiliative relationships between the group members. Second, three point behaviors regarding agonistic behavior were used: Contact Aggression (bite, pull, push, hit), Non-contact Aggression (display, chase), and Aggressive Displacement. These data were used to characterize the agonistic relationships between the group members.

In addition, during the focal observations the level of social vigilance of the focal individual was measured when another individual approached within $5 \mathrm{~m}$ of the focal individual (Watts, 1998), the focal individual was stationary, the approach was neutral and the focal individual was clearly visible for the observer. An approach was considered neutral if, (1) the approaching individual did not come from a direction where noise was produced just before his/her arrival (not applicable to subtle vocalizations, since these could not be heard by the observer), (2) the approaching individual approached at normal 
speed, and (3) the approaching individual was not directly followed by another individual. We used only neutral approaches to control for the fact that the behavior of the approaching individual during the approach would affect social vigilance.

The levels of vigilance were measured as ordinal response categories of the focal individual towards the approaching individual. The response categories were based on Watts (1998) and were categorized, from least intense to most intense, as: None ("focal does not look up at approaching subject"); Glance ("focal looks up at approaching subject for less than one second, without interrupting other behaviors"); Look ("focal gazes at approaching subject for more than one second, without interrupting other behavior and no change of posture, except for the head"); and Watch ("focal orients towards approaching subject and fixes her/his gaze on the approaching subject for more than one second and interrupts activity if applicable").

\section{Data Processing}

\section{Sociality Index (SI)}

We calculated a Sociality Index (SI; Silk et al., 2006) as a measure of dyadic affiliation. The Sociality Index was calculated on the basis of the affiliation data for each dyad $(N=91)$ and contained two behavioral terms: proximity or play $(\mathrm{P})$ and contact or allogrooming $(\mathrm{C})$. First, the SI contained the total proportion of time spent in proximity of or playing with the other individual $\left(\mathrm{P}_{\text {dyad }}\right)$. Social play and proximity were combined to prevent an age effect in the sociality index, because adult individuals spend almost no time on playing. For every dyad, the $\mathrm{P}_{\text {dyad }}$ was divided by the average $\mathrm{P}$ over all dyads $\left(\mathrm{P}_{\text {average }}\right)$. Second, it contained the total proportion of time spent in contact with or allogrooming of the other individual $\left(\mathrm{C}_{\text {dyad }}\right)$. Contact and allogrooming were taken together because allogrooming very rarely happened. For every dyad, the $\mathrm{C}_{\mathrm{dyad}}$ was divided by the average $\mathrm{C}$ over all dyads $\left(\mathrm{C}_{\text {average }}\right)$. Then, these two terms were added together for each dyad and divided by 2 (the number of terms).

Sociality Index $=\frac{\frac{P_{\text {dyad }}}{P_{\text {average }}}+\frac{C_{\text {dyad }}}{C_{\text {average }}}}{2}$

To compute the Sociality Index of a dyad, focal data of both individuals were combined. This resulted in an observation time of approximately $18 \mathrm{hr}$ per dyad, while the results for dyads involving Kisiwa (who was transferred during the study period) were based on approximately $8 \mathrm{hr}$ per dyad.

\section{Agonistic Data}

While previous studies on vigilance in despotic species have often used dominance rank in their analyses, we included dyadic aggression rates in our analyses, given that western lowland gorillas do not form linear dominance relationships and show no clear submission to other individuals than the silverback male (Scott \& Lockard, 1999; Stokes, 2004).

To analyze agonistic data per dyad, we summed the frequencies of all point behaviors in the categories Contact Aggression, Non-contact Aggression and Aggressive Displacement for both individuals separately per dyad. This resulted in the frequency of agonistic behaviors of both individuals within the dyad towards each other during the full observation period. Hereafter, we divided the frequencies by the total dyadic observation time to obtain an aggression rate per hour for both individuals within the dyad. 


\section{Age-Sex Class}

Age-sex class definitions followed Hutchinson and Fletcher (2010): 4- to 6-year-olds were categorized as infants, 6- to 8-year-old individuals were categorized as adolescents, and females older than 8 years or males older than 12 years were categorized as adults. In addition, 8- to 12-year-old males are generally categorized as blackbacks. However, two males in our study were in between adolescent and blackback stage during the observation period. Therefore, we refer to them as adolescents for convenience's sake.

\section{Data Analysis}

All data were analyzed using R Statistics version 3.5.0 (R Core Team, 2018) and RStudio version 1.1.453 (RStudio Team, 2016). All Bayesian models were created in Stan computational framework and accessed using the brms package (Bürkner, 2017, 2018), and we used ggplot2 (Wickham, 2009) for data visualization.

\section{Relationship Between Affiliation and Aggression}

To test whether dyadic aggression and affiliation were correlated, we used a Bayesian Mixed Effects Model. We log-transformed SI-values to make the variable more linear, and mean-centered the transformed value, using the following formula:

$S I_{\text {transformed }}=\log 10(S I+1)-\log 10(2)$

In our analysis, aggression rate between all possible dyads was the dependent variable (182 cases; 91 dyads*2) and transformed SI-values were the independent variable (population-level effect). In addition, we added random intercepts (group-level effects) for the IDs of both aggressor and aggressee to avoid pseudoreplication. Because the aggression-data contained many zeroes, we implemented a zeroinflated beta family for the model. We ran the model with four chains of 3,000 iterations each, half of which were warmups.

To improve convergence and guard against overfitting, we specified mildly informative conservative priors for the population-level effects (Gaussian distribution $M=0, S D=5$ ). After running the model, we used the WAMBS checklist (Depaoli \& van de Schoot, 2017) to diagnose possible problems related to model convergence. First, the trace plots exhibited convergence, which was also corroborated by the Gelman-Rubin diagnostics that were all close to 1 . Second, trace plots still exhibited convergence after doubling the number of iterations. Third, we obtained smooth histograms for all posterior distributions of the parameters. Fourth, we observed no excessive autocorrelation among samples.

We report the $95 \%$ credible interval $(95 \% \mathrm{CrI})$ for the posterior probability distribution of the population-level effect.

\section{Social Vigilance Data}

We analyzed social vigilance data using a Bayesian sequential model. This analysis is suitable for ordinal dependent variables and assumes that higher response categories are only possible after lower categories have been achieved. For a more extensive introduction to sequential models, please see Bürkner and Vuorre (2019).

In our model, the level of vigilance during each neutral approach was the dependent variable (ordinal). We included the following independent variables (population-level effects): (1) the meancentered log-transformed SI (see above), (2) the mean-centered aggression rate of the approaching individual towards the focal individual, and (3) the age-sex class of the approaching individual. We chose adult females as the reference category for our analysis because most individuals belonged to this age-sex 
class ( 5 in total). In addition, we included a random intercept for the ID of the approacher and the focal in the models (group-level effect). We ran the model with four chains of 3,000 iterations each, half of which were warmups.

We did not include matrilineal kinship in our model, since matrilineal kinship was strongly collinear with our measure of relationship quality (Appendix Figure A1). Dyads with high relationship quality were mostly -but not exclusively- related to each other. This is a common pattern among primates (e.g., Emery Thompson, 2019).

To improve convergence and guard against overfitting, we specified mildly informative conservative priors for the population-level effects (Gaussian distribution $M=0, S D=5$ ). After running the model, we used the WAMBS checklist (Depaoli \& van de Schoot, 2017) to diagnose possible problems related to model convergence. First, the trace plots exhibited convergence, which was also corroborated by the Gelman-Rubin diagnostics that were all (close to) 1 . Second, trace plots still exhibited convergence after doubling the number of iterations. Third, we obtained smooth histograms for all posterior distributions of the parameters. Fourth, we observed no excessive autocorrelation among samples. effects.

We report $95 \%$ credible intervals for the posterior probability distributions of the population-level

\section{Data Availability Statement}

The dataset and analysis code are available via https://osf.io/4tvfq/.

\section{Ethics Statement}

All applicable national and/or institutional guidelines for the care and use of animals were followed. The research concerned non-invasive observations that in no way intervened with the normal procedures and behavior of the animals.

\section{Results}

\section{Relationship Between Affiliation And Aggression}

First, we explored whether dyadic affiliation and dyadic aggression were correlated (Table 1). We found a negative correlation between aggression rate and affiliation (-0.37, 95\% CrI [-0.99, 0.23]; Figure 1 ), but this effect was not statistically robust as the credible interval contained 0 .

\section{Level of Vigilance During Neutral Approaches}

For all neutral approaches $(N=627)$ we analyzed the visual response of the focal individual. In total, 170 of the 182 possible focal-approacher combinations were observed ( 91 possible dyads*2=182). The visual responses to the 627 recorded neutral approaches were classified: 119 as 'none,' 267 as 'glance,' 205 as 'look,' and 36 as 'watch.' 
Roth \& Sterck 543

\section{Table 1}

Model Output for the Zero-Inflated Beta Model Measuring the Effect of Relationship Quality (Log-Transformed Sociality Index) On Aggression Rate

\begin{tabular}{lcc}
\hline Predictors & Aggression Estimates & CrI (95\%) \\
Intercept & -1.69 & $-1.92--1.48$ \\
logSIcentral & -0.37 & $-0.99-0.23$ \\
Random Effects & & \\
sd (Intercept) Aggressor & 0.15 & $0.01-0.41$ \\
sd (Intercept) Aggressee & 0.11 & $0.00-0.33$ \\
Observations & 182 & \\
$\mathrm{~N}_{\text {aggressor }}$ & 14 & \\
$\mathrm{~N}_{\text {aggressee }}$ & 14 & \\
\hline
\end{tabular}

Figure 1

Relationship Between Aggression and Relationship Quality.
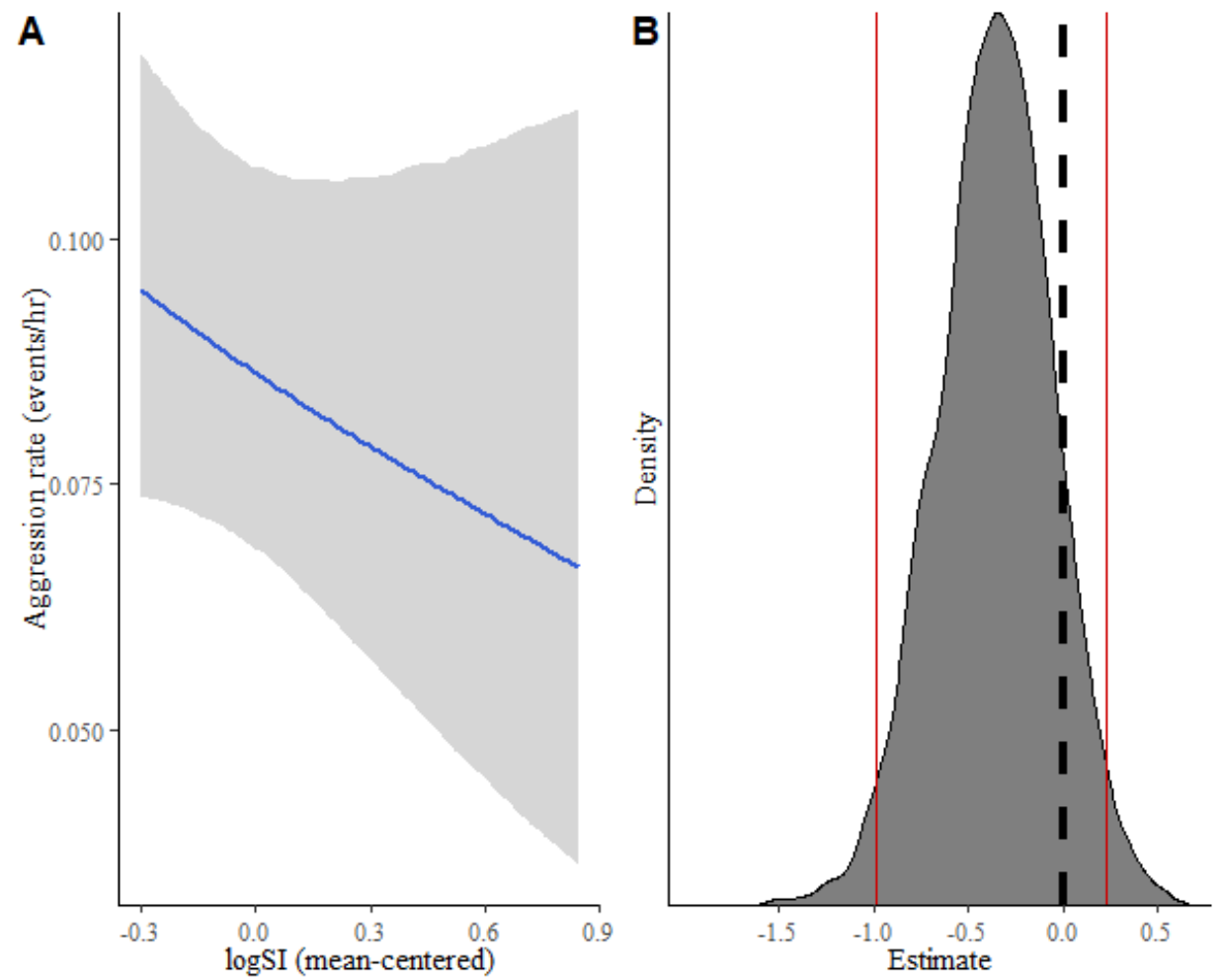

Note. (A) Correlation between aggression rate and the measure of relationship quality (Mean centered logSI); (B) Posterior density for the effect of Mean-centered logSI on aggression rate. Red lines show the $95 \% \mathrm{CrI}$. 


\section{Predictors of Social Vigilance}

The sequential model that we used to predict level of vigilance contained aggression rate (meancentered), logSI (mean-centered) and age-sex class of the approacher as independent variables (Table 2).

First, we found a statistically robust negative correlation between $\operatorname{logSI}$ and level of vigilance ($1.19,95 \%$ CrI [-1.75; -0.66]; Figure 2). This means that the probability of receiving a higher level of vigilance decreased when the approacher and the focal individual had a higher SI, indicative of an affiliative relationship.

\section{Table 2}

Model Output for the Sequential Model Measuring the Effect of Relationship Quality (Log-Transformed Sociality Index), Rate of Aggression and the Approacher's Age-Sex Class on the Level of Vigilance from the Approached Focal Individual

\begin{tabular}{lcc}
\hline & Level of vigilance & \\
\hline Predictors & Log-Odds & CrI (95\%) \\
logSIcentral & -1.19 & $-1.75--0.66$ \\
TotalAggressioncentral & 0.96 & $-0.29-2.16$ \\
ApproacherAgeSexAd.F & -0.64 & $-1.87-0.59$ \\
ApproacherAgeSexJ.F & -0.72 & $-1.55-0.05$ \\
ApproacherAgeSexA.M & 2.20 & $0.98-3.46$ \\
ApproacherAgeSexAd.M & 0.61 & $-0.30-1.45$ \\
ApproacherAgeSexJ.M & -1.19 & $-2.10--0.34$ \\
Random Effects & & \\
sd (Intercept) Looker & & $0.00-0.28$ \\
sd (Intercept) Approacher & 0.09 & $0.10-0.89$ \\
Observations & 0.42 & \\
$\mathrm{~N}_{\text {looker }}$ & 627 & \\
$\mathrm{~N}_{\text {approacher }}$ & 14 & \\
\hline
\end{tabular}

Second, we found a positive correlation between aggression rate and level of vigilance $(0.96,95 \%$ CrI [-0.29, 2.16]; Figure 3), but this effect was not statistically robust. The probability of the lowest levels of vigilance decreased when the aggression rate of approacher towards the focal individual was higher. The opposite effect was found for higher levels of vigilance.

Third, level of vigilance depended strongly on the age-sex class of the approaching individual. Especially the adult males, and to a lesser extent the adolescent males, received higher levels of vigilance during their approaches (Figure 4; Table 3). While all other age-sex classes were most likely to receive glances when they approached a conspecific, the adult male and adolescent males were most likely to receive looks upon their approaches. 


\section{Figure 2}

Effect of Relationship Quality on Level of Vigilance
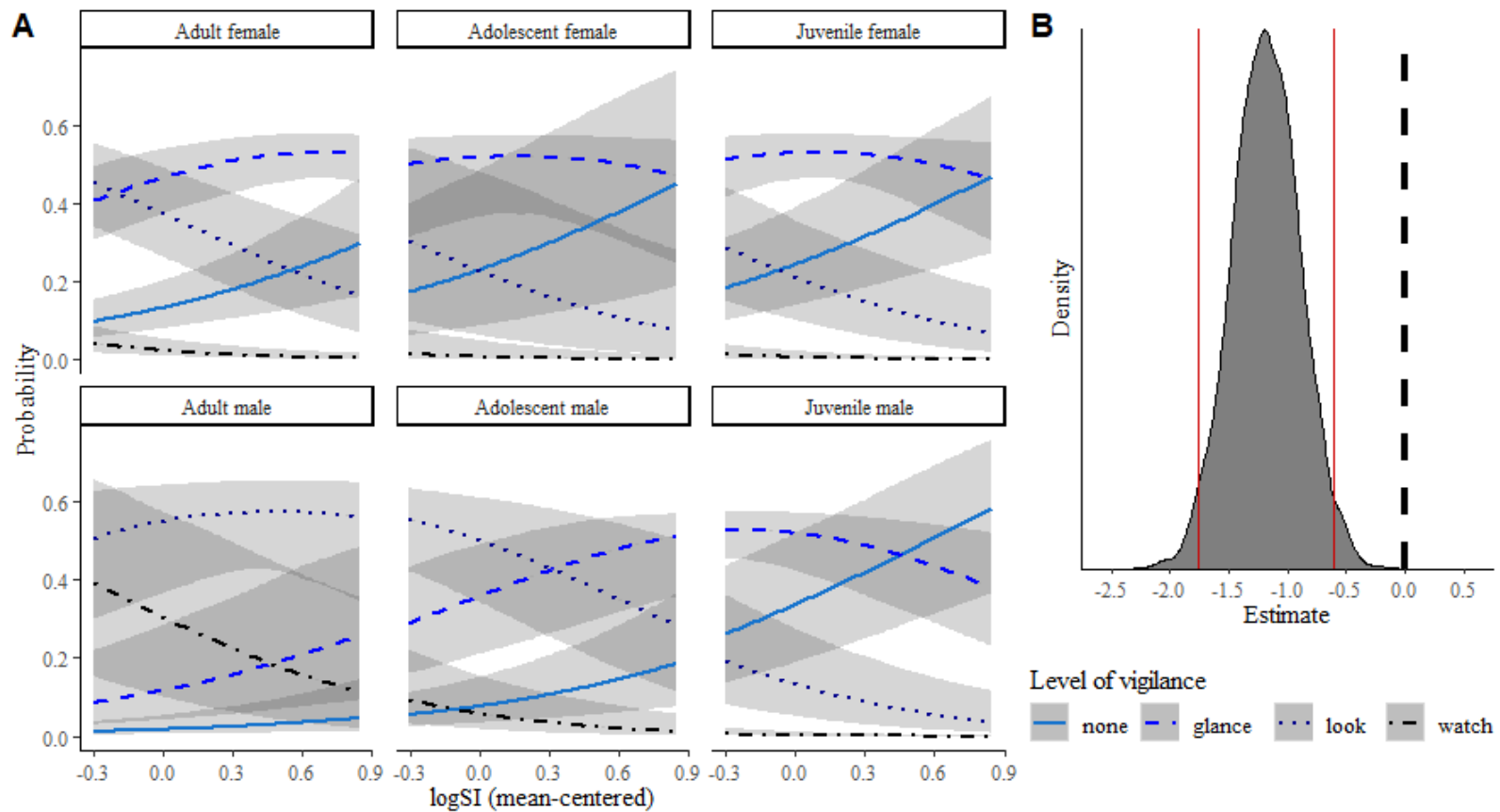

Level of vigilance

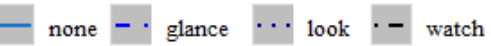

Note. (A) Correlation between the probability of each level of vigilance occurring and mean-centered logSI (measure of relationship quality) of the approacher and the focal individual per approacher age-sex class; (B) Posterior density for the effect of mean-centered $\operatorname{logSI}$ on level of vigilance. Red lines show the $95 \% \mathrm{CrI}$.

\section{Figure 3}

Effect of Aggression Rate on Level of Vigilance

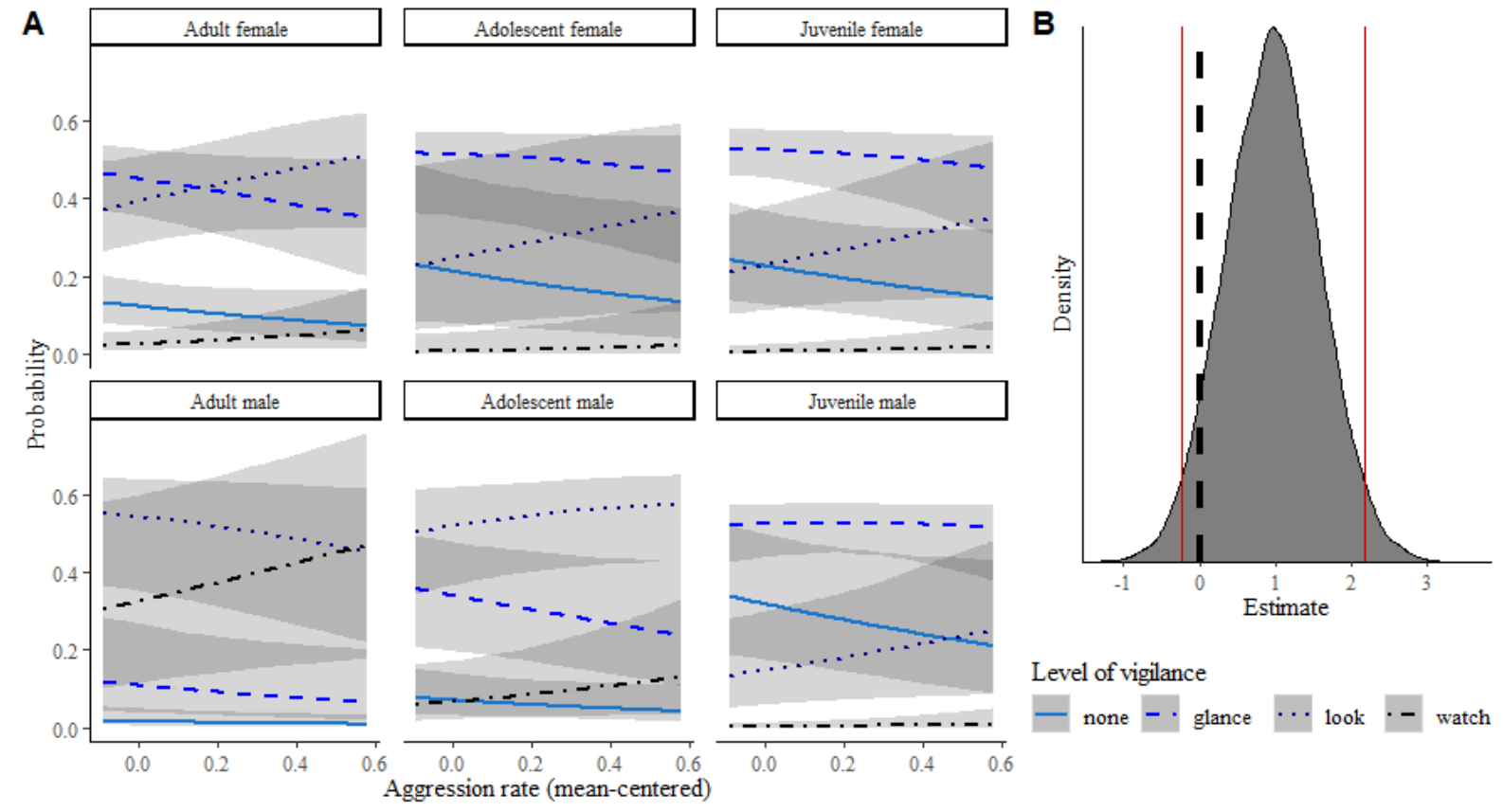

Note. (A) Correlation between the probability of each level of vigilance occurring and mean-centered aggression rate of the approacher towards the focal individual per approacher age-sex class; (B) Posterior density for the effect of mean-centered aggression rate on level of vigilance. Red lines show the $95 \% \mathrm{CrI}$. 


\section{Figure 4}

Effect of Age-Sex Class on Level of Vigilance
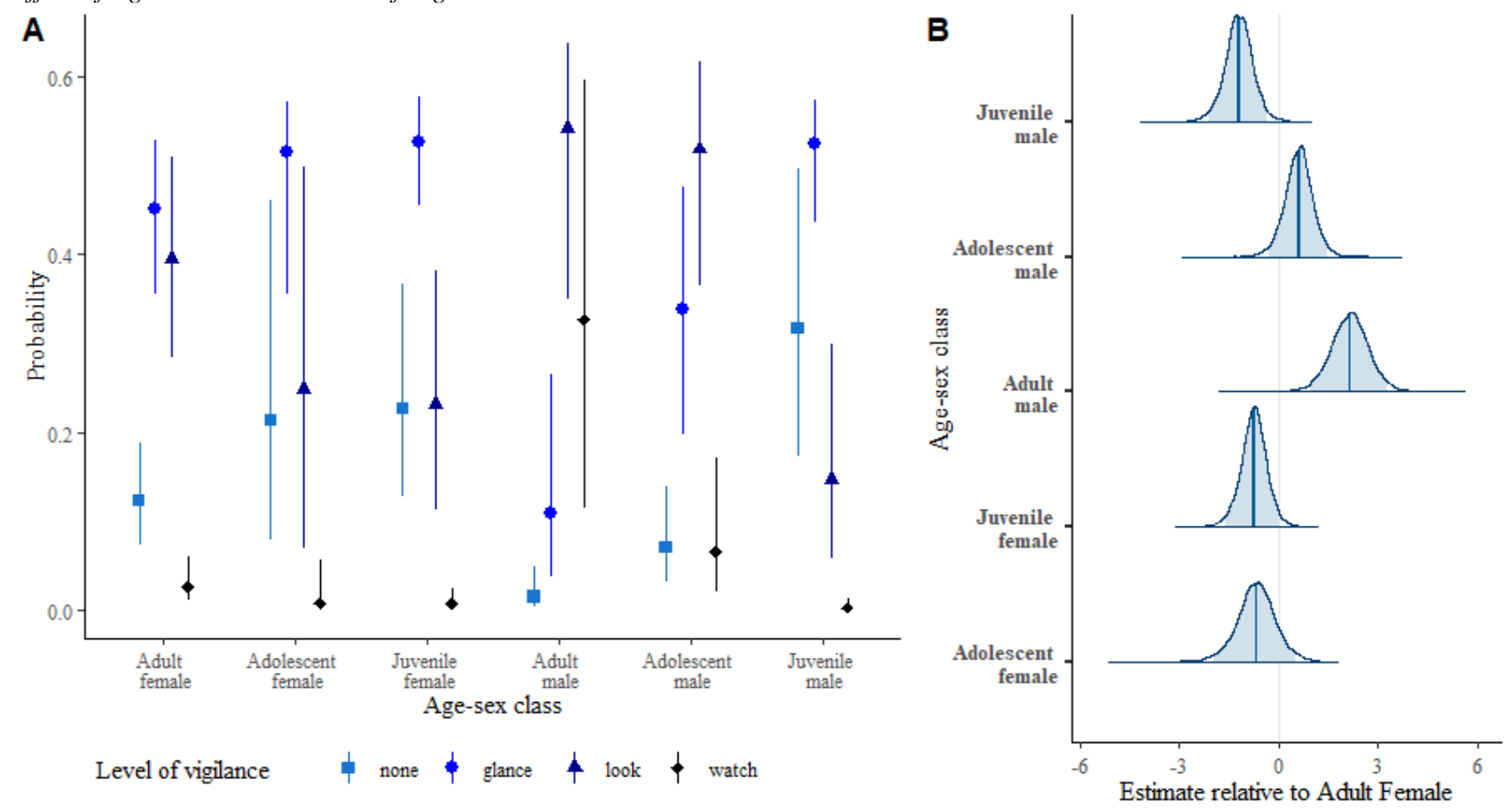

Note. (A) Probability of the different levels of vigilance per approacher age-sex class; (B) Estimates for each approacher age-sex class relative to the reference category (Adult Female). Negative values indicate that approachers from this age-sex class are more likely to receive lower levels of vigilance than adult females, while positive values indicate that approachers from this agesex class are more likely to receive higher levels of vigilance.

Table 3

Level of Vigilance During Approaches: Multiple Comparisons Between All Approacher Age-Sex Classes

\begin{tabular}{lccc}
\hline Comparison & Estimate & 95\% CrI (lower) & 95\% CrI (upper) \\
\hline Adolescent male - Adult female & 0.61 & 0.61 & -0.30 \\
Adult male - Adult female & 2.18 & 2.20 & 0.98 \\
Adolescent female - Adult female & -0.65 & -0.64 & -1.87 \\
Juvenile female - Adult female & -0.74 & -0.72 & -1.55 \\
Juvenile male - Adult female & -1.2 & -1.19 & -2.10 \\
Adolescent male - Adult male & -1.57 & -1.59 & -3.02 \\
Adolescent male - Juvenile male & 1.81 & 1.80 & 0.74 \\
Adolescent male - Adolescent female & 1.26 & 1.26 & -0.10 \\
Adolescent male - Juvenile female & 1.35 & 1.34 & 0.33 \\
Adult male - Juvenile male & 3.38 & 3.39 & 2.09 \\
Adult male - Adolescent female & 2.83 & 2.85 & 1.26 \\
Adult male - Juvenile female & 2.92 & 2.93 & 1.66 \\
Juvenile male - Adolescent female & -0.55 & -0.55 & -1.94 \\
Juvenile male - Juvenile female & -0.46 & -0.47 & -1.45 \\
Adolescent female - Juvenile female & 0.09 & 0.08 & -1.19 \\
\hline
\end{tabular}




\section{Discussion}

We investigated whether the level of social vigilance in reaction to approach of a conspecific is related to dyadic affiliation and aggression in a naturalistic family group of zoo-housed western lowland gorillas. Previous studies in primates have mainly focused on the effect of agonistic interactions on social vigilance and have found that social vigilance is often directed to aggressive individuals (e.g., Pannozzo et al., 2007). In contrast, a few studies have studied the effect of affiliative experiences on social vigilance and have found that good relationship quality can relax social vigilance (Kutsukake, 2006; Watts, 1998). Our results indicate that both aggressive and affiliative experiences affect social vigilance levels in western lowland gorillas, but that only the effect of dyadic affiliation is statistically robust. Here, we discuss the implications of our results, and address possible limitations of our study.

The differential effects of agonism and affiliation on social vigilance can only be distinguished when aggressive and affiliative behavior represent a different characteristic of social relationships and do not correlate strongly. We did find a negative effect of dyadic relationship quality on aggression rate, but this effect was not statistically robust. This matches results of other studies, that found no clear differences in aggression between individuals with affiliative and non-affiliative relationships (e.g., Bernstein \& Ehardt, 1986; Silk et al., 2010a). Therefore, it can be useful to address the effects of aggression and affiliation separately in studies of primate sociality (Overduin-de Vries et al., 2020; Vermande \& Sterck, in press).

Being vigilant towards aggressive individuals helps to prevent aggression by conspecifics. Accordingly, we found that focal individuals showed higher levels of vigilance in response to aggressive conspecifics, but this effect was not statistically robust. This might be the result of relatively the egalitarian relationships that female western lowland gorillas form, where the rate of aggression is low, aggression is often ignored (Stokes, 2004), and dominance hierarchies are nonlinear (Scott \& Lockard, 1999). However, western low-land gorilla male-female relationships are more consistent and unidirectional, with males being aggressive towards females (Stokes, 2004). This was reflected in the fact that the adult male and two adolescent males were monitored more intensively than other group members. As agonistic relationships between western lowland gorillas are not pronounced, except for male-female relationships, aggressive experiences may have a less strong effect on social vigilance when compared to more despotic species. Nevertheless, the direction of the effect was obvious, and we consider this result therefore in line with previous research on aggression and social vigilance (reviewed in Beauchamp, 2015).

Alternatively, males could be monitored more intensely as a result of their larger potential for aggression. The adult and adolescent males probably have most potential for aggression because they are physically the strongest individuals in the group. Having a conflict with one of those individuals would therefore be more likely to result in injuries. By being vigilant towards adult and adolescent males, individuals may therefore reduce the risk of being attacked by the individuals who could inflict most harm. This also raises the question whether social vigilance in gorillas is directed either at the individuals with whom a focal individual has had a lot of aggressive interactions, or the individuals that have the largest potential for aggression (e.g., the individuals with the largest body size). Of course, these two are not mutually exclusive. This remains to be further explored.

With regards to affiliative interactions, we found that individuals with a better relationship quality monitored each other less intensely. This is consistent with two studies on relatively egalitarian primates (chimpanzees: Kutsukake, 2006; mountain gorillas: Watts, 1998). Thus, our results indicate that social vigilance is influenced by negative interactions and by positive, affiliative interactions. This raises the question why positive affiliative interactions may relax social vigilance. We propose that aggressive interactions between individuals with an affiliative relationship have less detrimental effects than aggression between individuals with a non-affiliative relationship. First, individuals may be more tolerant towards conspecifics with whom they have an affiliative relationship (Silk et al., 2010a; Watts, 1994). Moreover, their aggression may be less likely to escalate (Watts, 1994), and individuals with an affiliative 
relationship reconcile more easily in many primate species (e.g., Aureli et al., 1997; Bernstein, 1991; Cords \& Aureli 2000; Watts, 2006). Note, however, that this seems not to apply to western lowland gorillas (Mallavarapu et al., 2006; Watts, 1995). Lastly, individuals with a good relationship might have more secure, predictable interactions (Cords \& Aureli, 2000), thereby reducing the need for intensive monitoring. Concluding, individuals can avoid escalated aggression by monitoring non-affiliative individuals, while this is not necessary for individuals with whom they have an affiliative relationship.

It is important to note that kinship and the dyadic sociality index were strongly related in this study and it was impossible to determine whether it is the affiliative relationships resulting in less intense monitoring or the underlying kinship. Interestingly, a recent study on monitoring quantity of mandrills found that individuals spent significantly more time monitoring kin (Schino \& Sciarretta, 2016), seemingly contradicting the findings of this study on monitoring intensity. However, how monitoring quantity (as in the mandrill study) and intensity of social vigilance (this study) relate to one another remains to be established, indicating the need for consistency in the study of primate vigilance (Allan \& Hill, 2018). Altogether, the relative effect of affiliative relationships and kinship on social vigilance requires more attention.

Thus far, most studies linked social vigilance to aggression (Beauchamp, 2015) and have not studied the effect of affiliative relationships. The species in which an effect of affiliative relationships on social vigilance has been found are typically relatively tolerant and egalitarian species. It is unclear whether such social vigilance will also be found in more despotic species. We argued that social vigilance may serve to monitor less affiliative, and therefore less predictable, individuals. In addition, receiving aggression from non-affiliative individuals may be more harmful. This should also apply to despotic primates. In these species, the rate of aggression from kin and affiliated group members is relatively high (baboons: Silk et al., 2010a; macaques: Bernstein \& Ehardt, 1986). However, aggression from familiar group members is reconciled more often than from unfamiliar group members (Aureli et al., 1997). This predicts that despotic primates will also benefit from social vigilance towards non-affiliative individuals.

It is important to note that our study has three important limitations. First, we only observed one family group, which means that we are not sure whether our results will extend to other western lowland gorilla groups. Additionally, the small sample size means that some age-sex classes were represented by only one or two individuals. We found a significant effect of age-sex class of the approacher on the level of vigilance. Because of the low sample size, it remains to be established if this is really an age-sex class effect, or if it is the result of differences in personality. For example, displacement behavior (mountain gorilla: Eckardt et al., 2015) and aggression (western lowland gorilla: Schaefer \& Steklis, 2014) are related to personality. Therefore, it will be necessary to increase the sample size per age-sex class. Second, we could not account for the role of subtle vocalizations during approaches, because it was not possible to hear the subtle sounds through the enclosure glass. Western lowland gorillas produce a wide array of vocalizations (Lemasson et al., 2018; Salmi et al., 2013). If gorillas produce calls during approaching, this may influence social vigilance. In accordance with this expectation, Watts (1998) found that female mountain gorillas were more vigilant when an approaching individual produced a mildly aggressive vocalization. This shows that social vigilance can be influenced by vocalizations. Third, the western lowland gorillas were housed in a relatively small space when compared to their outdoor enclosure. A previous study in the same zoo reported multiple behavioral changes associated with being housed in the indoor enclosure, such as larger interindividual distances and more avoidance. Importantly, frequency of conflicts was not one of them (Cordoni \& Palagi, 2007). Nevertheless, it is important to note that housing circumstances could have enhanced social vigilance. Therefore, future studies could try to corroborate our findings in a more natural setting.

In conclusion, captive western lowland gorillas showed higher levels of vigilance for individuals with whom they had more agonistic and less affiliative experiences. This effect was robust for affiliative experiences, but only marginal for aggressive experiences. This pattern may fit relatively egalitarian species like the western lowland gorilla, where the rate of aggression is low and aggression is often ignored. In despotic species, monitoring high risk individuals may be more important. However, also 
these primates show variation in dyadic affiliation that has an effect on fitness (Massen et al., 2010; Overduin-de Vries et al., 2020; Seyfarth \& Cheney, 2012). Therefore, also in these species affiliative experiences might explain variation in social vigilance.

\section{Acknowledgements}

We thank Apenheul Primate Park, and in particular Thomas Bionda and the animal caretakers, for their support of this study. Furthermore, we thank two anonymous reviewers for their feedback on earlier versions of the manuscript.

\section{References}

Allan, A. T. L., \& Hill, R. A. (2018). What have we been looking at? A call for consistency in studies of primate vigilance. American Journal of Physical Anthropology, 165, 4-22. https://doi.org/10.1002/ajpa.23381

Altmann, J. (1974). Observational study of behavior: Sampling methods. Behaviour, 49, 227-266. https://doi.org/10.1163/156853974x00534

Aureli, F. (1992). Post-conflict behaviour among wild long-tailed macaques (Macaca fascicularis). Behavioral Ecology and Sociobiology, 31, 329-337. https://doi.org/10.1007/bf00177773

Aureli, F., Cords, M., \& van Schaik, C. P. (2002). Conflict resolution following aggression in gregarious animals: A predictive framework. Animal Behaviour, 64, 325-343. https://doi.org/10.1006/anbe.2002.3071

Aureli, F., Das, M., \& Veenema, H. C. (1997). Differential kinship effect on reconciliation in three species of macaques (Macaca fascicularis, M. fuscata, and M. sylvanus). Journal of Comparative Psychology, 111, 91-99. https://doi.org/10.1037/0735-7036.111.1.91

Beauchamp, G. (2015). Animal vigilance: Monitoring predators and competitors. Academic Press. https://doi.org/10.1016/C2014-0-01423-2

Bernstein, I. S. (1991). The correlation between kinship and behaviour in non-human primates. In P. G. Hepper (Ed.), Kin recognition (pp. 6-29). Cambridge University Press.

Bernstein, I. S., \& Ehardt, C. (1986). The influence of kinship and socialization on aggressive behaviour in rhesus monkeys (Macaca mulatta). Animal Behaviour, 34, 739-747. https://doi.org/10.1016/s0003$\underline{3472(86) 80057-4}$

Bettridge, C. M., \& Dunbar, R. I. M. (2012). Predation as a determinant of minimum group size in baboons. Folia Primatologica, 83, 332-352. https://doi.org/10.1159/000339808

Bürkner, P.-C. (2017). brms: An R package for Bayesian multilevel models using Stan. Journal of Statistical Software, 80(1). https://doi.org/10.18637/jss.v080.i01

Bürkner, P.-C. (2018). Advanced Bayesian multilevel modeling with the R package brms. The R Journal, 10, 395411. https://doi.org/10.32614/rj-2018-017

Bürkner, P.-C., \& Vuorre, M. (2019). Ordinal regression models in psychology: A tutorial. Advances in Methods and Practices in Psychological Science, 2, 77-101. https://doi.org/10.1177/2515245918823199

Cameron, E. Z., Setsaas, T. H., \& Linklater, W. L. (2009). Social bonds between unrelated females increase reproductive success in feral horses. Proceedings of the National Academy of Sciences, 106, 13850-13853. https://doi.org/10.1073/pnas.0900639106

Chalyan, V. G., \& Meishvili, N. V. (2007). Aggression and the composition of conflicting pairs in green monkeys. Neuroscience and Behavioral Physiology, 37, 915-919. https://doi.org/10.1007/s11055-007-0099-z

Connor, R. C. (2007). Dolphin social intelligence: Complex alliance relationships in bottlenose dolphins and a consideration of selective environments for extreme brain size evolution in mammals. Philosophical Transactions of the Royal Society B: Biological Sciences, 362, 587-602. https://doi.org/10.1098/rstb.2006.1997

Cordoni, G., \& Palagi, E. (2007). Response of captive lowland gorillas (Gorilla gorilla gorilla) to different housing conditions: Testing the aggression-density and coping models. Journal of Comparative Psychology, 121, 171-180. https://doi.org/10.1037/0735-7036.121.2.171

Cords, M., \& Aureli, F. (2000). Reconciliation and relationship qualities. In F. Aureli \& F. B. M. de Waal (Eds.), Natural conflict resolution (pp. 177-198). University of California Press.

de Waal, F. B. M. (2000). Primates--A natural heritage of conflict resolution. Science, 289, 586-590. https://doi.org/10.1126/science.289.5479.586 
Depaoli, S., \& van de Schoot, R. (2017). Improving transparency and replication in Bayesian statistics: The WAMBS-Checklist. Psychological Methods, 22, 240-261. https://doi.org/10.1037/met0000065

Drews, C. (1996). Contexts and patterns of injuries in free-ranging male baboons (Papio cynocephalus). Behaviour, 133, 443-474. https://doi.org/10.1163/156853996x00530

Eckardt, W., Steklis, H. D., Steklis, N. G., Fletcher, A. W., Stoinski, T. S., \& Weiss, A. (2015). Personality dimensions and their behavioral correlates in wild Virunga mountain gorillas (Gorilla beringei beringei). Journal of Comparative Psychology, 129, 26-41. https://doi.org/10.1037/a0038370

Elgar, M. A. (1989). Predator vigilance and group size in mammals and birds: A critical review of the empirical evidence. Biological Reviews 64, 13-33. https://doi.org/10.1111/j.1469-185X.1989.tb00636.x

Ellis, S., Franks, D. W., Nattrass, S., Cant, M. A., Weiss, M. N., Giles, D., Balcomb, K. C., \& Croft, D. P. (2017). Mortality risk and social network position in resident killer whales: Sex differences and the importance of resource abundance. Proceedings of the Royal Society B: Biological Sciences, 284, 20171313. https://doi.org/10.1098/rspb.2017.1313

Ellis, S., Snyder-Mackler, N., Ruiz-Lambides, A., Platt, M. L., \& Brent, L. J. N. (2019). Deconstructing sociality: The types of social connections that predict longevity in a group-living primate. Proceedings of the Royal Society B: Biological Sciences, 286, 20191991. https://doi.org/10.1098/rspb.2019.1991

Emery Thompson, M. (2019). How can non-human primates inform evolutionary perspectives on female-biased kinship in humans? Philosophical Transactions of the Royal Society B: Biological Sciences, 374, 20180074. https://doi.org/10.1098/rstb.2018.0074

Evers, E., de Vries, H., Spruijt, B. M., \& Sterck, E. H. M. (2012). Look before you leap - Individual variation in social vigilance shapes socio-spatial group properties in an agent-based model. Behavioral Ecology and Sociobiology, 66, 931-945. https://doi.org/10.1007/s00265-012-1342-3

Gaynor, K. M., \& Cords, M. (2012). Antipredator and social monitoring functions of vigilance behaviour in blue monkeys. Animal Behaviour, 84, 531-537. https://doi.org/10.1016/j.anbehav.2012.06.003

Gosselin-ildari, A. D., \& Koenig, A. (2012). The effects of group size and reproductive status on vigilance in captive Callithrix jacchus. American Journal of Primatology, 74, 613-621. https://doi.org/10.1002/ajp.22013

Hutchinson, J. E., \& Fletcher, A. W. (2010). Using behavior to determine immature life-stages in captive western gorillas. American Journal of Primatology, 72, 492-501. https://doi.org/10.1002/ajp.20799

Keverne, E. B., Leonard, R. A., Scruton, D. M., \& Young, S. K. (1978). Visual monitoring in social groups of talapoin monkeys (Miopithecus talapoin). Animal Behaviour, 26, 933-944. https://doi.org/10.1016/0003$\underline{3472(78) 90157-4}$

Kutsukake, N. (2006). The context and quality of social relationships Affect vigilance behaviour in wild chimpanzees. Ethology, 112, 581-591. https://doi.org/10.1111/j.1439-0310.2006.01200.x

Lemasson, A., Pereira, H., \& Levréro, F. (2018). Social basis of vocal interactions in western lowland gorillas (Gorilla g. gorilla). Journal of Comparative Psychology, 132, 141-151. https://doi.org/10.1037/com0000105

Less, E. H., Lukas, K. E., Kuhar, C. W., \& Stoinski, T. S. (2010). Behavioral response of captive western lowland gorillas (Gorilla gorilla gorilla) to the death of silverbacks in multi-male groups. Zoo Biology, 29, 16-29. https://doi.org/10.1002/zoo.20246

Maestripieri, D., Schino, G., Aureli, F., \& Troisi, A. (1992). A modest proposal: Displacement activities as an indicator of emotions in primates. Animal Behaviour, 44, 967-979. https://doi.org/10.1016/s0003$\underline{3472(05) 80592-5}$

Magliocca, F., Querouil, S., \& Gautier-Hion, A. (1999). Population structure and group composition of western lowland gorillas in North-Western Republic of Congo. American Journal of Primatology, 48, 1-14. https://doi.org/10.1002/(sici)1098-2345(1999)48:1<1::aid-ajp1>3.0.co;2-2

Mallavarapu, S., Stoinski, T. S., Bloomsmith, M. A., \& Maple, T. L. (2006). Postconflict behavior in captive western lowland gorillas (Gorilla gorilla gorilla). American Journal of Primatology, 68, 789-801. https://doi.org/10.1002/ajp.20279

Massen, J., Sterck, E., \& de Vos, H. (2010). Close social associations in animals and humans: Functions and mechanisms of friendship. Behaviour, 147, 1379-1412. https://doi.org/10.1163/000579510x528224

McNelis, N. L., \& Boatright-Horowitz, S. L. (1998). Social monitoring in a primate group: The relationship between visual attention and hierarchical ranks. Animal Cognition, 1, 65-69. https://doi.org/10.1007/s100710050008

Noldus, L. P. J. J. (1991). The Observer: A software system for collection and analysis of observational data. Behavior Research Methods, Instruments, \& Computers, 23, 415-429. https://doi.org/10.3758/bf03203406 
Overduin-de Vries, A. M., de Vries, H., Vermande, M. M., Reijntjes, A. H. A., \& Sterck, E. H. M. (2020). Both aggressive and affiliative behaviour facilitate resource access in high-ranking female long-tailed macaques (Macaca fascicularis). Behaviour, 157, 267-287. https://doi.org/10.1163/1568539x-bja10001

Pannozzo, P. L., Phillips, K. A., Haas, M. E., \& Mintz, E. M. (2007). Social monitoring reflects dominance relationships in a small captive group of brown capuchin monkeys (Cebus apella). Ethology, 113, 881-888. https://doi.org/10.1111/j.1439-0310.2007.01392.x

R Core Team. (2018). R: A language and environment for statistical computing. R Foundation for Statistical Computing.

RStudio Team. (2016). RStudio: Integrated development for $R$. RStudio Inc.

Salmi, R., Hammerschmidt, K., \& Doran-Sheehy, D. M. (2013). Western gorilla vocal repertoire and contextual use of vocalizations. Ethology, 119, 831-847. https://doi.org/10.1111/eth.12122

Sarfaty, A., Margulis, S. W., \& Atsalis, S. (2012). Effects of combination birth control on estrous behavior in captive western lowland gorillas, Gorilla gorilla gorilla. Zoo Biology, 31, 350-361. https://doi.org/10.1002/zoo.20401

Schaefer, S. A., \& Steklis, H. D. (2014). Personality and subjective well-being in captive male western lowland gorillas living in bachelor groups. American Journal of Primatology, 76, 879-889. https://doi.org/10.1002/ajp.22275

Schino, G., \& Sciarretta, M. (2016). Patterns of social attention in mandrills, Mandrillus sphinx. International Journal of Primatology, 37, 752-761. https://doi.org/10.1007/s10764-016-9936-7

Scott, J., \& Lockard, J. (1999). Female dominance relationships among captive western lowland gorillas: Comparisons with the wild. Behaviour, 136, 1283-1310. https://doi.org/10.1163/156853999500721

Seyfarth, R. M., \& Cheney, D. L. (2012). The evolutionary origins of friendship. Annual Review of Psychology, 63, 153-177. https://doi.org/10.1146/annurev-psych-120710-100337

Silk, J. B. (2002). Kin selection in primate groups. International Journal of Primatology, 23, 849-875. https://doi.org/10.1023/A:1015581016205

Silk, J. B. (2003). Social bonds of female baboons enhance infant survival. Science, 302, $1231-1234$. https://doi.org/10.1126/science. 1088580

Silk, J. B., Altmann, J., \& Alberts, S. C. (2006). Social relationships among adult female baboons (Papio cynocephalus) I. Variation in the strength of social bonds. Behavioral Ecology and Sociobiology, 61, 183195. https://doi.org/10.1007/s00265-006-0249-2

Silk, J. B., Beehner, J. C., Bergman, T. J., Crockford, C., Engh, A. L., Moscovice, L. R., Wittig, R. M., Seyfarth, R. M., \& Cheney, D. L. (2010a). Female chacma baboons form strong, equitable, and enduring social bonds. Behavioral Ecology and Sociobiology, 64, 1733-1747. https://doi.org/10.1007/s00265-010-0986-0

Silk, J. B., Beehner, J. C., Bergman, T. J., Crockford, C., Engh, A. L., Moscovice, L. R., Wittig, R. M., Seyfarth, R. M., \& Cheney, D. L. (2010b). Strong and consistent social bonds enhance the longevity of female baboons. Current Biology, 20, 1359-1361. https://doi.org/10.1016/j.cub.2010.05.067

Stokes, E. J. (2004). Within-group social relationships among females and adult males in wild western lowland gorillas (Gorilla gorilla gorilla). American Journal of Primatology, 64, $233-246$. https://doi.org/10.1002/ajp.20074

Sussman, R. W., Garber, P. A., \& Cheverud, J. M. (2005). Importance of cooperation and affiliation in the evolution of primate sociality. American Journal of Physical Anthropology, $128 \quad 84-97$. https://doi.org/10.1002/ajpa.20196

Thompson, N. A. (2019). Understanding the links between social ties and fitness over the life cycle in primates. Behaviour, 156, 859-908. https://doi.org/10.1163/1568539x-00003552

Treves, A. (2000). Theory and method in studies of vigilance and aggregation. Animal Behaviour, 60, $711-722$. https://doi.org/10.1006/anbe.2000.1528

van Schaik, C. P. (1983). Why are diurnal primates living in groups? Behaviour, 87, $120-144$. https://doi.org/10.1163/156853983x00147

van Schaik, C. P., \& van Noordwijk, M. A. (1985). Evolutionary effect of the absence of felids on the social organization of the macaques on the island of Simeulue (Macaca fascicularis fusca, Miller 1903). Folia Primatologica, 44, 138-147. https://doi.org/10.1159/000156208

Vermande, M. M. \& Sterck, E. H. M. (in press) How to get the biggest slice of the cake. A comparative view of social behaviour and resource access in human children and non-human primates. Frontiers in Psychology/Education. https://doi.org/10.3389/fpsyg.2020.584815 
Watts, D. P. (1994). Social relationships of immigrant and resident female mountain gorillas, II: Relatedness, residence, and relationships between females. American Journal of Primatology, 32, 13-30. https://doi.org/10.1002/ajp.1350320103

Watts, D. P. (1995). Post-conflict social events in wild mountain gorillas (Mammalia, Hominoidea). I. Social interactions between opponents. Ethology, 100, 139-157. https://doi.org/10.1111/j.14390310.1995.tb00321.x

Watts, D. P. (1998). A preliminary study of selective visual attention in female mountain gorillas (Gorilla gorilla beringei). Primates, 39, 71-78. https://doi.org/10.1007/bf02557744

Watts, D. P. (2006). Conflict resolution in chimpanzees and the valuable-relationships hypothesis. International Journal of Primatology, 27, 1337-1364. https://doi.org/10.1007/s10764-006-9081-9

Wickham, H. (2009). ggplot2. Springer. https://doi.org/10.1007/978-0-387-98141-3

Widdig, A., Bercovitch, F., Nürnberg, P., Streich, W. J., \& Krawczak, M. (2002). Affiliation and aggression among adult female rhesus macaques: A genetic analysis of paternal cohorts. Behaviour, 139, 371-391. https://doi.org/10.1163/156853902760102717 


\section{Appendix}

\section{Table A1}

Characteristics of Study Subjects and Total Observation Time

\begin{tabular}{|l|l|l|l|l|l|}
\hline Individual & Birth & Sex & Age class & Matriline & Observation \\
\hline Jambo & 1993 & Male & Adult & - & $09: 00: 00$ \\
\hline Mintha & 1974 & Female & Adult & Mintha & $08: 59: 01$ \\
\hline Manji & 1975 & Female & Adult & Manji & $09: 00: 00$ \\
\hline Kisiwa* & 1997 & Female & Adult & Dalila & $04: 00: 00$ \\
\hline Nemsi & 2001 & Female & Adult & Manji & $08: 58: 50$ \\
\hline Gyasi & 2002 & Female & Adult & Dalila & $08: 54: 08$ \\
\hline Mapasa & 2007 & Male & Adolescent/Blackback & Manji** & $08: 58: 44$ \\
\hline Wimbe & 2008 & Male & Adolescent/Blackback & Lobo & $08: 57: 50$ \\
\hline M'Fugaji & 2009 & Female & Adolescent & Mintha & $08: 50: 37$ \\
\hline Iriki & 2011 & Female & Juvenile & Manji & $08: 54: 53$ \\
\hline Chama & 2011 & Female & Juvenile & Dalila & $08: 57: 24$ \\
\hline Tayari & 2011 & Female & Juvenile & Dalila & $08: 56: 28$ \\
\hline M'Zungu & 2011 & Male & Juvenile & Manji & $08: 53: 39$ \\
\hline Jabari & 2012 & Male & Juvenile & Lobo & $08: 56: 55$ \\
\hline
\end{tabular}

Note. $*=$ Kisiwa was relocated to another zoo after 4 weeks of observation.

**= Mapasa's birth mother is Kisiwa, but he was adopted by Manji directly after birth. 
Table A2

Ethogram for This Study

\begin{tabular}{|c|c|c|c|}
\hline Category & Behavior & Type & Definition \\
\hline \multirow[t]{8}{*}{ Time budget } & Foraging & Duration & Searching for, handling or consuming food or water. \\
\hline & Inactive & Duration & Sitting, lying down or hanging, including sleeping. \\
\hline & Move & Duration & $\begin{array}{l}\text { Moving: walking/ running, not directed towards or from } \\
\text { another individual. }\end{array}$ \\
\hline & Social play & Duration & $\begin{array}{l}\text { Two or more animals play with each other, play face is } \\
\text { visible. }\end{array}$ \\
\hline & Non-social play & Duration & $\begin{array}{l}\text { Toying with own body, not directed at other animals or } \\
\text { handling, examining or manipulating objects. }\end{array}$ \\
\hline & Auto-grooming & Duration & $\begin{array}{l}\text { Manipulating of own hair or skin with hand, foot or } \\
\text { mouth. }\end{array}$ \\
\hline & Allo-grooming & Duration & $\begin{array}{l}\text { Searching through hair or skin of another individual with } \\
\text { hands or mouth and manipulating it. }\end{array}$ \\
\hline & Other & Duration & $\begin{array}{l}\text { Timebudget behaviors that do not fall into either of the } \\
\text { aforementioned categories (i.e. nest building). }\end{array}$ \\
\hline \multirow[t]{2}{*}{ Affiliation } & Proximity & Duration & $\begin{array}{l}\text { Being in the direct vicinity: less than one meter away, of } \\
\text { one or more individuals, in a non-agonistic and non- } \\
\text { sexual manner. }\end{array}$ \\
\hline & Contact & Duration & $\begin{array}{l}\text { Sitting next to or on another individual with physical } \\
\text { contact. }\end{array}$ \\
\hline \multirow[t]{4}{*}{ Contact aggression } & Bite & Event & $\begin{array}{l}\text { Using the mouth/teeth in an aggressive way on the body } \\
\text { of another. }\end{array}$ \\
\hline & Hit & Event & Slap another with hands or feet with force. \\
\hline & Push & Event & Shove another individual with force. \\
\hline & Pull & Event & Grabbing body part of another while exerting force. \\
\hline \multirow[t]{2}{*}{$\begin{array}{l}\text { Non-contact } \\
\text { aggression }\end{array}$} & Display & Event & $\begin{array}{l}\text { Dominant body posture (such as quadrupedal stance or } \\
\text { standing solely on hind legs, sometimes with chest } \\
\text { beating), exposing of teeth (canines) and/or aggressively } \\
\text { striking object. }\end{array}$ \\
\hline & Chase & Event & $\begin{array}{l}\text { Animal pursues another and follows the fleeing } \\
\text { individual at high speed. }\end{array}$ \\
\hline $\begin{array}{l}\text { Aggressive } \\
\text { displacement }\end{array}$ & Aggressive displace & Event & $\begin{array}{l}\text { Aggressive approach, at high speed, of another } \\
\text { individual (within reach) resulting in the withdrawal of } \\
\text { the other individual. }\end{array}$ \\
\hline \multirow[t]{2}{*}{ Submission } & Hide & Event & $\begin{array}{l}\text { Staying out of the sight of one or more (aggressive) } \\
\text { individual(s) }\end{array}$ \\
\hline & Flee & Event & $\begin{array}{l}\text { Run at high speed to get away from (an)other } \\
\text { (aggressive) individual(s). }\end{array}$ \\
\hline $\begin{array}{l}\text { Non-aggressive } \\
\text { displacement }\end{array}$ & $\begin{array}{l}\text { Non-aggressive } \\
\text { displacement }\end{array}$ & Event & $\begin{array}{l}\text { Non-aggressive approach, at normal speed, of another } \\
\text { individual (within reach) resulting in the withdrawal of } \\
\text { the other individual. }\end{array}$ \\
\hline \multirow[t]{5}{*}{ Social attention } & $\begin{array}{l}\text { Neutral } \\
\text { appearance }\end{array}$ & Event & $\begin{array}{l}\text { Approach of another individual within 3-5 meters of the } \\
\text { focal individual, when the focal individual is stationary. } \\
\text { Only scored if the approach is neutral. }\end{array}$ \\
\hline & None & Event & Individual does not look up at approaching subject. \\
\hline & Glance & Event & $\begin{array}{l}\text { Individual gazes at approaching subject for less than one } \\
\text { second, without interrupting other behaviors. }\end{array}$ \\
\hline & Look & Event & $\begin{array}{l}\text { Individual gazes at approaching subject for more than } \\
\text { one second, without interrupting other behavior and no } \\
\text { change of posture, except for the head. }\end{array}$ \\
\hline & Watch & Event & $\begin{array}{l}\text { Individual orients towards approaching subject and fixes } \\
\text { her/his gaze on the approaching subject for more than } \\
\text { one second and interrupts activity (if applicable). }\end{array}$ \\
\hline
\end{tabular}


Roth \& Sterck 555

Figure A1

Boxplot Depicting the Effect of Kinship on Relationship Quality (Measures as Mean-Centered logSI). K=Kin, NK=Nonkin

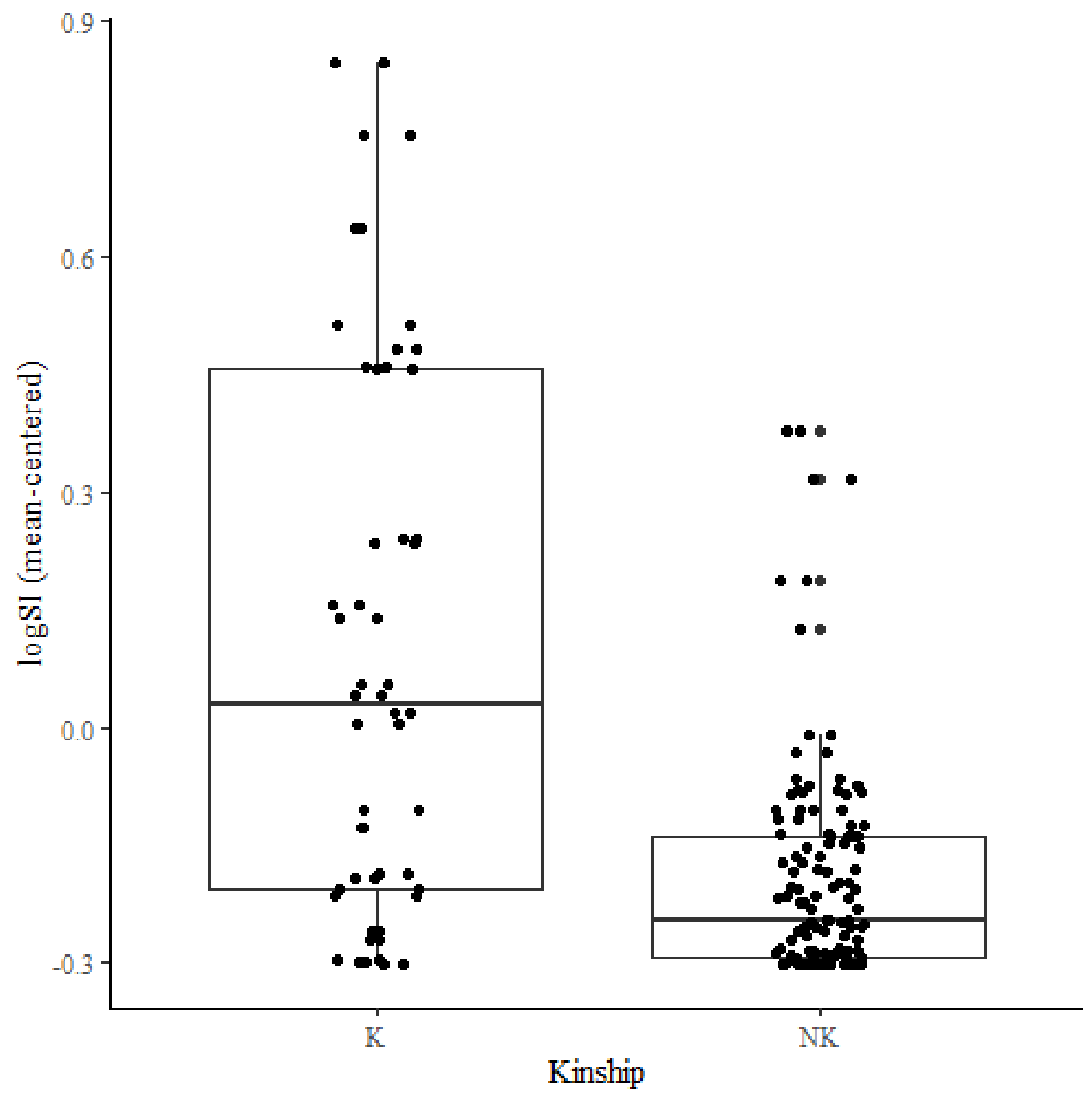

